\title{
LIST OF POSTERS PRESENTED AT THE JD15
}

Hereafter is the list of all the posters presented at the JD 15. For each poster, we present the list of author (with the email address of the first authors only) followed by the full title of their contribution.

JD15-005P: Kirn S.S. (sskim@uju.es.pusan.ac.kr), Lee H.M., Two-Component Fokker-Planck Models for Postcollapse Evolution of Globular Clusters.

JD15-006P: Funato Y. (funato@chianti.c.u-tokyo.ac.jp), Makino J., McMillan S., Hut P., An Universal Meta-Algorithm to Symmetrize Any One-step Time-integration scheme.

JD15-007P: Ishibashi N. (nobuo@info.kanagawa-u.ac.jp), Atuko N.,

A High Accuracy Numerical Method for Solving a Set of Ordinary Differential Equations with Spline Functions.

JD15-008P: Fukushige T. (fukushig@chianti.c.u-tokyo.ac.jp), Makino J., Taiji M., Highly-Parallelized Special-Purpose Computer for N-body Simulations: GRAPE-5.

JD15-009P: Kawai A. (kawai@chianti.c.u-tokyo.ac.jp), Fukushige T., Taiji M., Makino J., Sugimoto D.,

The PCI interface for GRAPE systems: PCI-HIB.

JD15-013P: DeMarchi G. (demarchi@eso.org), Paresce F., The IMF of Low-Mass Stars in Globular Clusters.

JD15-016P: Chen W.P. (wchen@phyast.phy.ncu.edu.tw), Kao K.C., Hu J.Y., Luminosity Function Evolution of Young Star Clusters.

JD15-020P: Fridman A.M. (afridman@inasan.rssi.ru):

Determination of the 3D Distribution Function in Stellar Clusters.

JD15-021P: Leon S. (sleon@mesunb.obspm.fr), Combes F., Meylan G., Leeuwin F., Disk-shocking on Globular Clusters: NGC $5139 \equiv \omega$ Centauri.

JD15-022P: Einsel C. (crwe@ari.uni-heidelberg.de), Spurzem R, On the Rotation of Early Globular Clusters.

JD15-023P: Rosenberg A. (alf@obelix.pd.astro.it), Piotto G., Saviane I., Aparicio A., An Homogeneous Photometric VI Data Base of GGCs (Progress Report).

JD15-029P: Nordstroem B. (birgitta@astro.ku.dk), Andersen J., Stellar and Dynamical Evolution in the Open Cluster NGC 3680.

JD15-030P: Rey S.C. (screy@galaxy.yonsei.ac.kr), Lee Y.W., Chun M.S., Byun Y.I., Blue Straggler Stars in the Globular Cluster M53.

JD15-031P: Klessen R. (klessen@mpia-hd.mpg.de), Burkert A.,

Fragmentation of Molecular Clouds with GRAPESPH: The Initial Phase of a Stellar Cluster.

JD15-032P: Piotto G. (piotto@astrpd.pd.astro.it), Sosin C., Djorgovski S., King I.R., Rich R.M., Dorman B., Renzini A., Phinney E.S., Liebert J., Meylan G., HST Observations of High-Density Globular Clusters.

JD15-036P: Anosova J. (anosova@clyde.as.utexas.edu), Benedict G.F., Dynamical Explorations of Nuclear Structures in Barred Galaxies.

JD15-038P: Just A. (just@ari.uni-heidelberg.de), Fuchs B., Wielen R., Self-consistent Vertical Profile of the Galactic Disk. 
JD15-039P: Fridman A.M. (afridman@inasan.rssi.ru), Lyakhovich V.V., Khoruzhii O.V., Silchenko O.K., Zasov A.V., Afanas'iev V.L., Dodonov S.N., Boulesteix J.,

Spiral - Vortex Structure in Gaseous Disks of Galaxies NGC 157, NGC 3893, NGC 6181, NGC 1365.

JD15-040P: Fridman A.M. (afridman@inasan.rssi.ru), Khoruzhii O.V.,

Nonlinear Radial Laminated Flow as a Manifestation of 3-D Dynamics of Astrophysical Disks.

JD15-042P: Vesperini E. (vesperin@falcon.phast.umass.edu),

On the Evolution of the Mass Function of the Galactic Globular Cluster System.

JD15-043P: Deng L. (licai@qso.bao.ac.cn), Chen R., Chen J.S.,

Observations of Galactic Clusters Using the BAO Schmidt and BATC Filters.

JD15-LateP1: Chakraborty D.K. (dkc@atria.ernet.in), Sahu D.K., Thakur P., Dynamical Modeling of some Elliptical galaxies.

JD15-LateP2: Yuasa M. (yuasa@cc.kindai.ac.jp), Unno W., Magono S., Distance Determination of Mass-Losing Stars.

JD15-LateP3: Park H.S. (hspark@knuecc-sun.knue.ac.kr), Kim H., Kim J.T., Lee J.S., Analysis of the Second-Order Extinction Coefficient on CCD Photometry of M3.

JD15-LateP4: Baumgardt H. (holger@ari.uni-heidelberg.de), Wielen R., Evolution of Globular Cluster Systems in Galaxies. 